\title{
Modeling Infection of Strawberry Flowers by Botrytis cinerea Using Field Data
}

\author{
Xiangming Xu, David C. Harris, and Angela M. Berrie
}

Horticulture Research International-East Malling, West Malling, Kent ME19 6BJ, U.K.

Accepted for publication 6 September 2000.

\begin{abstract}
Xu, X.-M., Harris, D. C., and Berrie, A. M. 2000. Modeling infection of strawberry flowers by Botrytis cinerea using field data. Phytopathology 90:1367-1374.

The incidence of strawberry flower infection by Botrytis cinerea was monitored in unsprayed field plots in three successive years together with meteorological data and numbers of conidia in the air. There were large differences in conidia numbers and weather conditions in the 3 years. Three sets of models were derived to relate inoculum and weather conditions to the incidence of flower infection; by inoculum only, by weather variables only, and by both inoculum and weather variables. All the models fitted the observed incidence satisfactorily. High inoculum led to

more infection. Models using weather variables only gave more accurate predictions than models using inoculum only. Models using both weather variables and inoculum gave the best predictions, but the improvement over the models based on weather variables only was small. The relationship between incidence of flower infection and inoculum and weather variables was generally consistent between years. Of the weather variables examined, daytime vapor pressure deficit and nighttime temperature had the greatest effect in determining daily incidence of flower infection. Infection was favored by low day vapor pressure deficit and high night temperature. The accuracy and consistency of the weatherbased models suggest they could be explored to assist in management of gray mold.
\end{abstract}

Gray mold, caused by Botrytis cinerea Pers.:Fr., is one of the most important airborne diseases of the strawberry and can seriously reduce yield and postharvest quality. The disease results mainly from latent infection of flower parts, which develops into rot once fruit begins to ripen $(1,9,11,14,17)$. The open flower, white bud, and senescent flower stages are most susceptible to infection (14), whereas flowers at the green bud stage are relatively resistant $(9,14)$. Infections at the white bud stage, however, are much less likely to lead to gray mold than infections at the open flower stage (14). The incidence of gray mold correlates strongly with environmental variables during the flowering period, particularly long periods of high relative humidity $(12,22)$. Mathematical models have been developed to relate the incidence of petal and stamen necrosis and incidence of flower infection to temperature and wetness duration (4). The conditions required for infection of ripe fruit are not as stringent as for infection of flower parts $(3,8)$.

Gray mold is managed principally by protecting flowers from infection. Using biocontrol agents to manage Botrytis spp. has received much attention in the last decade $(6,7,18,19)$. Control of gray mold in commercial strawberry production, however, still relies heavily on the routine application of fungicides during the flowering and early fruiting periods. This is usually effective, but under conditions particularly conducive to disease, control may be less than satisfactory. Furthermore, the routine application of fungicides, irrespective of whether conditions are conducive to disease development, has become increasingly unacceptable. An alternative approach is to restrict fungicide application to periods conducive to disease development. A prerequisite for this approach is a disease forecasting system based on environmental conditions. A simple forecasting system for gray mold has been developed (8) from data obtained in controlled environments

Corresponding author: X.-M. Xu; E-mail address: xiangming.xu@hri.ac.uk

Publication no. P-2000-1010-02R

(C) 2000 The American Phytopathological Society
$(3,4)$, but the performance of this system and the effects of variable inoculum concentrations on disease development under field conditions are unknown.

The objective of this study was to develop mathematical models that relate the incidence of flower infection to inoculum concentration and weather conditions in the field, from which a disease forecasting system could be developed.

\section{MATERIALS AND METHODS}

Plants. Experiments were conducted on 1- or 2-year-old fieldgrown plants of the June bearing (short-day) 'Elsanta' over a period of 3 years (1995 to 1997). In each year, the experimental plot contained about 700 plants and consisted of six double rows on raised beds. The beds were covered with blue polythene mulch and were provided with trickle irrigation.

Plants were subjected to a full spray program for controlling pests, weeds, and diseases such as powdery mildew, except that all sprays for gray mold were omitted. No attempts were made to remove dead leaves from plants, although runners were cut from plants and transferred to alleys between the beds in autumn to early winter and killed by spraying with paraquat.

Data collection. The incidence of flower infection in each successive 2-day period during flowering was determined in each year. Ten flowers at the white-bud stage from each row were labeled, giving a total of 120 flowers, at about 0800 on day 1 . After $48 \mathrm{~h}$, these flowers were collected individually into 25-ml universal bottles, and a new set of 120 flowers at the white-bud stage was labeled as before. This process was repeated over the entire flowering period, i.e., 29 April to 28 May 1995, 9 May to 7 June 1996, and 11 April to 12 May 1997.

The flowers collected on each sampling date were surface-sterilized with sodium hypochlorite $(0.025 \%$ available chlorine, $\mathrm{wt} / \mathrm{vol}$ ) for $15 \mathrm{~min}$ to remove any spores on the surface and then rinsed with distilled water. The flowers were placed separately on filter paper thoroughly wetted with distilled water in small, sterile petri dishes. The dishes were incubated in a glasshouse compart- 
ment at approximately $20^{\circ} \mathrm{C}$ for 7 or 8 days, after which the flowers were examined for conidiophores of $B$. cinerea. Any flower on which conidiophores were detected was classified as infected.

A volumetric spore trap (Burkard Manufacturing Co. Ltd., Rickmansworth, Hertfordshire, UK) was used to sample air continuously within the plot at approximately 10 liters/min throughout the experimental periods. The daily numbers of conidia of $B$. cinerea in the air were estimated by scanning one transect of the tape at $\times 400$. The amount and duration of rainfall, duration of surface wetness, shade temperature, and ambient and in-canopy relative humidity $(r h)$ were recorded at 12 -min-intervals by an automatic weather station (Metos, Gottfried Pessl, Weiz, Austria) located in the plot. Preliminary results showed that in-canopy humidity was highly correlated with ambient humidity, thus only ambient humidity was used for analysis. Water vapor pressure $(v p)$ was derived from temperature using the following empirical equation:

$$
v p=4.6698 e^{0.06241 \mathrm{temp}}
$$

This equation was derived from the listed values for water vapor pressure and temperature (temp) (15) and accounted for about $99 \%$ of the total variation. Values of vapor pressure deficit $(v p d)$ were then calculated as

$$
v p d=v p(1-r h / 100)
$$

Statistical analysis. The daily number of conidia and corresponding weather variables are time series, characterized by temporal autocorrelation. Transfer function models were used to analyze the effects of weather variables on the number of conidia. Transfer function (TF) models are an extension of ordinary regres-
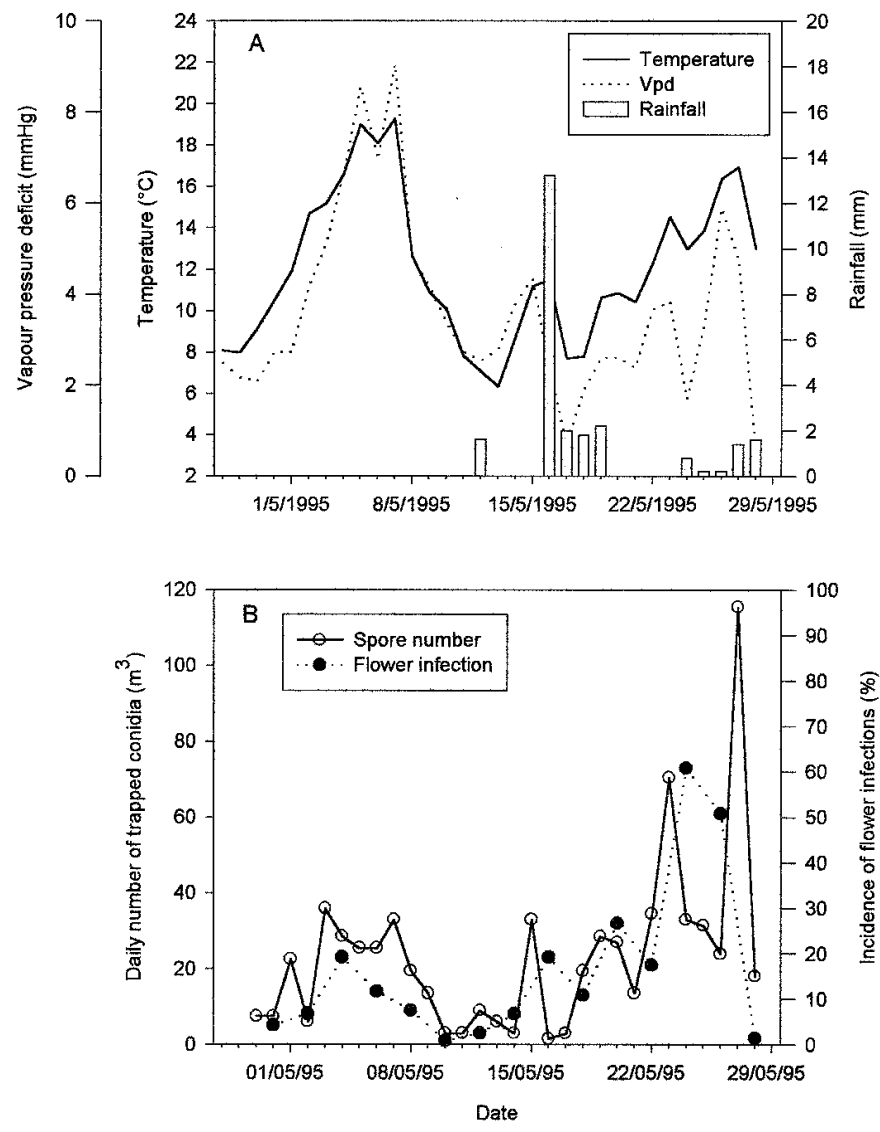

Fig. 1. Weather conditions and observed incidence of flower infection in 1995. A, Daily average vapor pressure deficit, daily average temperature, and daily total rainfall. B, Daily number of trapped conidia of Botrytis cinerea and observed incidence of infection of strawberry flowers in each 48-h-period. sion models (20) that recognize temporal dependence within both the dependent and independent variables. The assumption of the random component is an important difference between TF models and ordinary regression models. In ordinary regression, successive values of the random component are assumed to be independent, whereas in TF models these values are assumed to follow an autoregressive integrated moving average (ARIMA) model.

A straight regression of the incidence of flower infection in each 48-h period on corresponding averages of weather variables and conidia number was not appropriate for two reasons. First, a simple regression analysis assumed that the effects of weather on day $t$ and $t+1$ on the infection of flowers by conidia on day $t$ and $t+1$ were the same, which was not true. For example, weather variables on day $t$ had no direct effects on infection by conidia on day $t+1$. Second, this simple analysis ignores potential reinfections of the same flowers in 2 days. To surmount these shortcomings, the following method was used to model the effects of daily weather variables on the incidence of flower infection.

Daily incidence of infection $(D I I)$, i.e., the proportion of flowers infected during each day ( $24 \mathrm{~h}$ from 0800 to 0759 the following day), was logit transformed $(\ln [D I I /(1-D I I)])$ and assumed to be a function of daily weather and inoculum variables. We assumed weather variables determine the proportion of spores that would successfully infect flowers, giving the model

$$
\ln \left[D I I_{t} /\left(1-D I I_{t}\right)\right]=i_{t} \times f\left(w_{t}\right)
$$

where $w_{t}$ and $i_{t}$ were daily weather and inoculum variables on day $t$, respectively.

The recorded incidence of infection over a 48 -h period $\left(p_{t}\right.$ on flowers tagged on day $t$ and collected on day $t+2$ ), as a proportion of the total number of flowers recorded, was assumed to re-
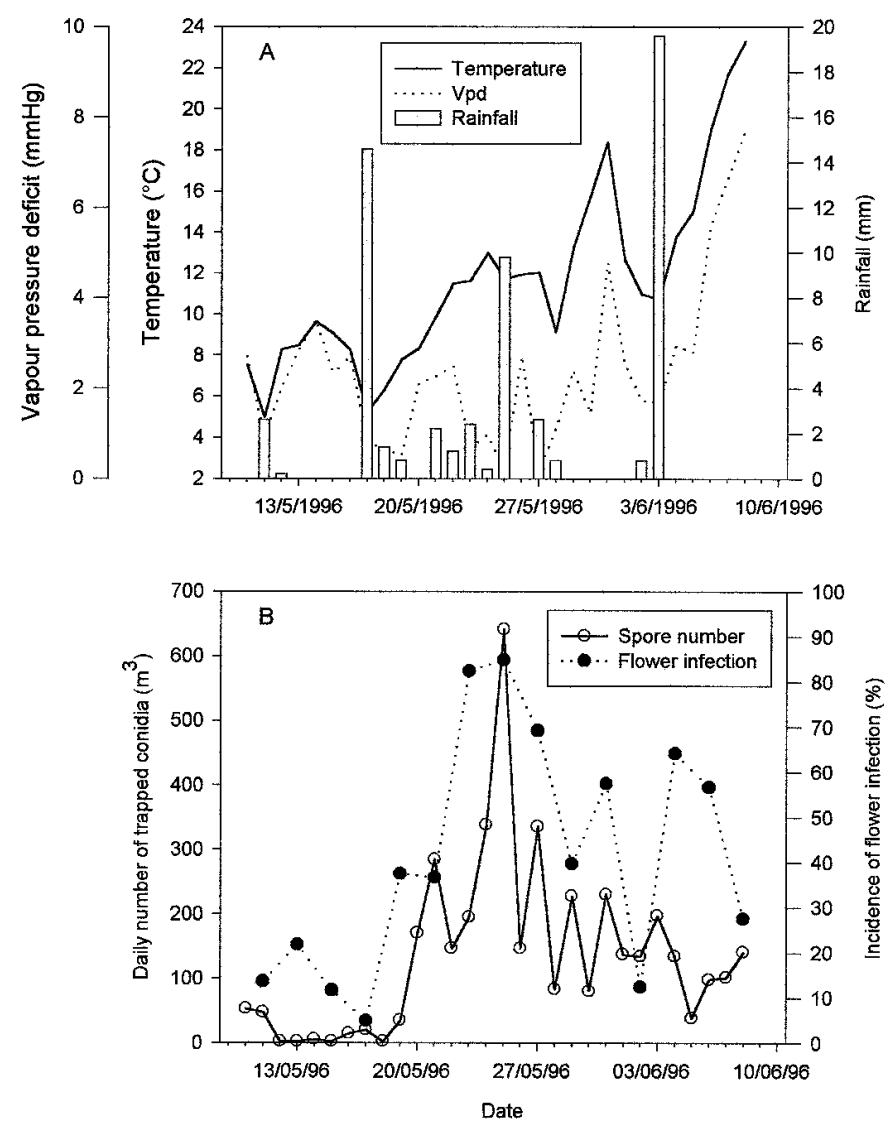

Fig. 2. Weather conditions and the observed incidence of flower infection in 1996. A, Daily average vapor pressure deficit, daily average temperature, and daily total rainfall. B, Daily number of trapped conidia of Botrytis cinerea and the observed incidence of infection of strawberry flowers in each 48-h period. 
sult from infection by conidia released on day $t$ and $t+1$ only. Because most conidia of $B$. cinerea are released between early morning and early afternoon (10), the success of infection by conidia released on day $t$ was assumed not to be affected by weather conditions on day $t+1$. Assuming that infection of flowers on successive days was independent, $p_{t}$ (incidence of flower infection during day $t$ and $t+1$ ) was related to $D I I_{t}$ and $D I I_{t+1}$ as follows:

$$
p_{t}=D I I_{t+1} \times\left(1-D I I_{t}\right)+D I I_{t}
$$

where DII is derived from equation (3) as

$$
D I I_{t}=\frac{\exp \left[i_{t} \times f\left(w_{t}\right)\right]}{1+\exp \left[i_{t} \times f\left(w_{t}\right)\right]}
$$

Thus

$$
\begin{aligned}
p_{t}= & \frac{\exp \left[i_{t} \times f\left(w_{t}\right)\right]}{1+\exp \left[i_{t} \times f\left(w_{t}\right)\right]}+\frac{\exp \left[i_{t+1} \times f\left(w_{t+1}\right)\right]}{1+\exp \left[i_{t+1} \times f\left(w_{t+1}\right)\right]} \times \\
& \frac{1}{1+\exp \left[i_{t} \times f\left(w_{t}\right)\right]}
\end{aligned}
$$

The DII was the response variable of interest, but was not directly observable from the recorded incidence of flower infections $\left(p_{t}\right)$ and was instead estimated indirectly based on equation 6. Equation 6, however, can be interpreted on the basis of DII under the two assumptions described above, i.e., daily infection was determined by current-day weather only, and infection of flowers on successive days was independent. Therefore, in this paper, to simplify model presentation all the regression results were pre-
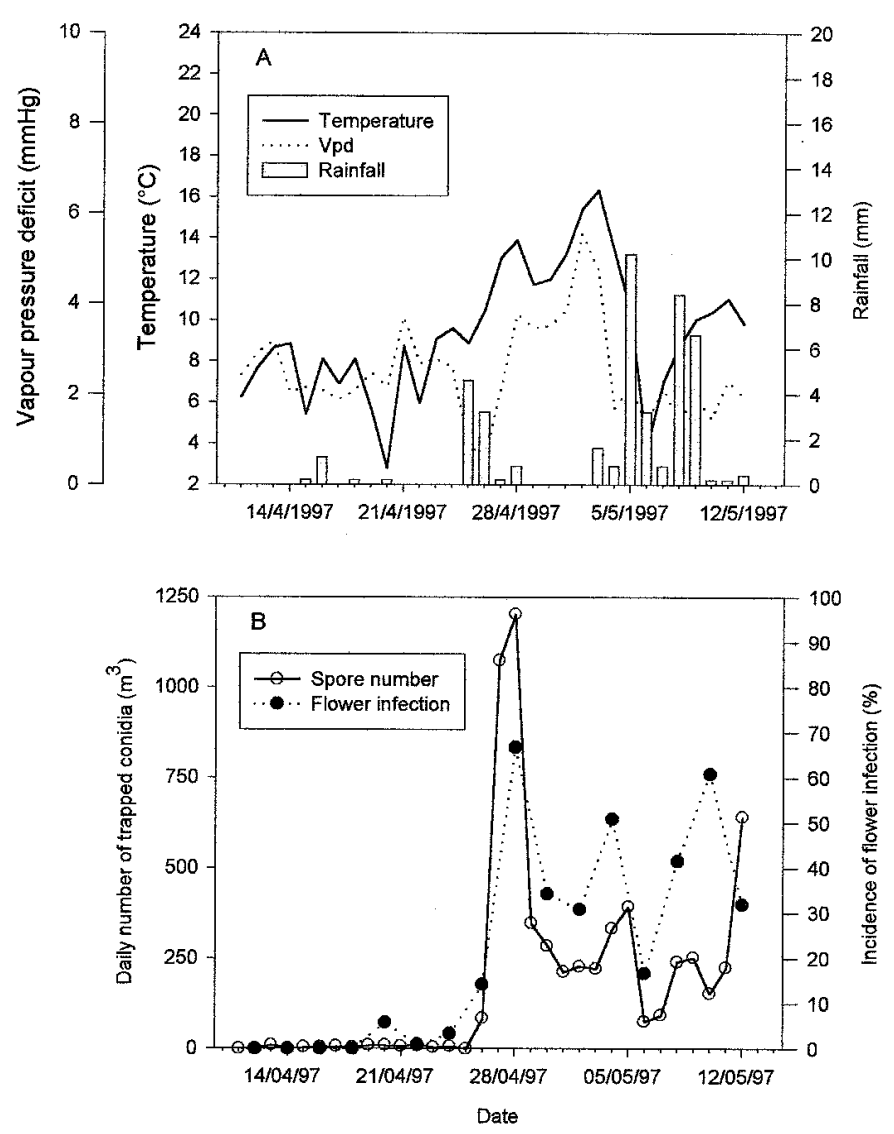

Fig. 3. Weather conditions and the observed incidence of flower infection in 1997. A, Daily average vapor pressure deficit, daily average temperature, and daily total rainfall. B, Daily numbers of trapped conidia of Botrytis cinerea and the observed incidence of infection of strawberry flowers in each 48-hperiod. sented in the form of DII (i.e., equation 3) instead of $p$ (equation 6) without explicitly stating the two assumptions.

Equation 6 was fitted using the FITNONLINEAR procedure, which estimates parameters using the maximum likelihood method (Genstat, Numerical Algorithms Group Ltd., Oxford) using a binomial model. The three sets of independent (explanatory) variables were: inoculum only, weather only, and inoculum and weather together. Weather variables included in the regression analysis were: temperature (degrees Celcius), relative humidity, amount (millimeters) and duration (hours) of rainfall, vapor pressure deficit $(v p d)$ (millimeters of mercury [mm $\mathrm{Hg}]$ or $\approx 133.3$ pascals), and duration of surface wetness (hours). For each of the weather variables, day (0800 to 1959) and night (2000 to 0759 the next day) averages as well as daily (0800 to 0759 the next day) averages were used in the regression analysis. Daily numbers of conidia trapped were transformed to natural logarithms. Because of the large differences in the numbers of conidia in the 3 years, the logarithm-transformed conidia numbers were then expressed as a percentage of the maximum in each year, and thus, the inoculum variable was expressed on the same scale (0 to $100 \%$ ) for the 3 years.

\section{RESULTS}

Correlation between numbers of conidia and weather variables. Daily numbers of conidia varied greatly from year to year (Figs. 1 through 3). Average daily numbers of conidia were 23, 116, and 192 for 1995, 1996, and 1997, respectively. There were large variations in the daily number of conidia within each year (Figs. 1 through 3), e.g., ranging from 0 to 1,203 in 1997.

There were significant positive correlations between conidia number and temperature in 1995 and 1997, and between conidia number and $r h$ in 1996 (Table 1). There was a significant negative correlation between conidia numbers and total duration of surface wetness on the previous day and on the same day (wet) in 1995. No other correlations were significant.

TABLE 1. Correlation between daily number of Botrytis cinerea conidia trapped and daily weather variables, 1995 to $1997^{\circ}$

\begin{tabular}{lccccc}
\hline Year & Temperature & Rainfall & Wet & Rainfall $_{t-1}{ }^{\mathrm{b}}$ & Wet $_{t-1}^{\mathrm{c}}$ \\
\hline 1995 & $0.53^{* *}$ & -0.14 & $-0.45^{*}$ & -0.17 & $-0.43^{*}$ \\
1996 & 0.25 & 0.28 & 0.08 & -0.09 & -0.25 \\
1997 & $0.55^{* *}$ & 0.08 & -0.19 & 0.06 & 0.19 \\
\hline
\end{tabular}

a $*$ and $* *$ indicate significance at the 1 and $5 \%$ levels, respectively (28 df except $199730 \mathrm{df}$ )

b Total rainfall $(\mathrm{mm})$ on the previous day

c Total duration of surface wetness on the previous day (hour)

TABLE 2. Multiple regression analyses of the incidence of Botrytis cinerea infection of strawberry flowers as a function of inoculum (relative daily number of conidia trapped) ${ }^{\mathrm{a}}$

\begin{tabular}{lcccc}
\hline & & Model 1 & & Model 2 \\
\cline { 5 - 5 } Term & Parameter & Common & & $(1995+1997)$ vs 1996 \\
\hline Constant $(1995+1997)$ & $b$ & & & $-6.30 \pm 0.441$ \\
Constant $(1996)$ & $b$ & $-5.71 \pm 0.443$ & & $-3.64 \pm 0.773$ \\
Inoculum $(1995+1997)$ & $a$ & & & $5.72 \pm 0.615$ \\
Inoculum (1996) & $a$ & $5.36 \pm 0.607$ & & $3.38 \pm 1.03$ \\
$\quad$ Variation accounted (\%) & & 63.5 & & 72.4 \\
$\quad$ Correlation between fitted & & 0.74 & \\
$\quad$ and observed & & & \\
\hline
\end{tabular}

${ }^{a}$ Under the two assumptions (daily infection was determined by current day weather only and infection of flowers on successive days was independent), regression results of equation 6 can be interpreted on the basis of daily incidence of infection (DII) (equation 3). Thus, the logit of the incidence of daily flower infection $\left[\operatorname{logit}\left(D I I_{t}\right)\right]$ was a function of daily inoculum, i.e. $\ln \left(D I I_{t} / 1-D I I_{t}\right)=a i_{t}+b$ where $a$ and $b$ are parameters. Model 1 had common parameter values for $a$ and $b$ for the 3 years whereas the parameters $a$ and $b$ for 1996 differed from those in the other 2 years in model 2 . 
Annual incidence of flower infections and weather. Most $B$. cinerea infections were found in flower petals. The average incidence of infection was highest in 1996 (41\%) and approximately $22 \%$ in 1995 and 1997 (Figs. 1 through 3). The incidence of flower infection in 48-h periods varied greatly within each year, e.g., ranging from 5.0 to $84.9 \%$ in 1996 .

Weather conditions differed considerably between years, as illustrated by temperature, vpd, and rainfall in Figures 1 through 3 . For example, the average daily temperature was lowest in 1997
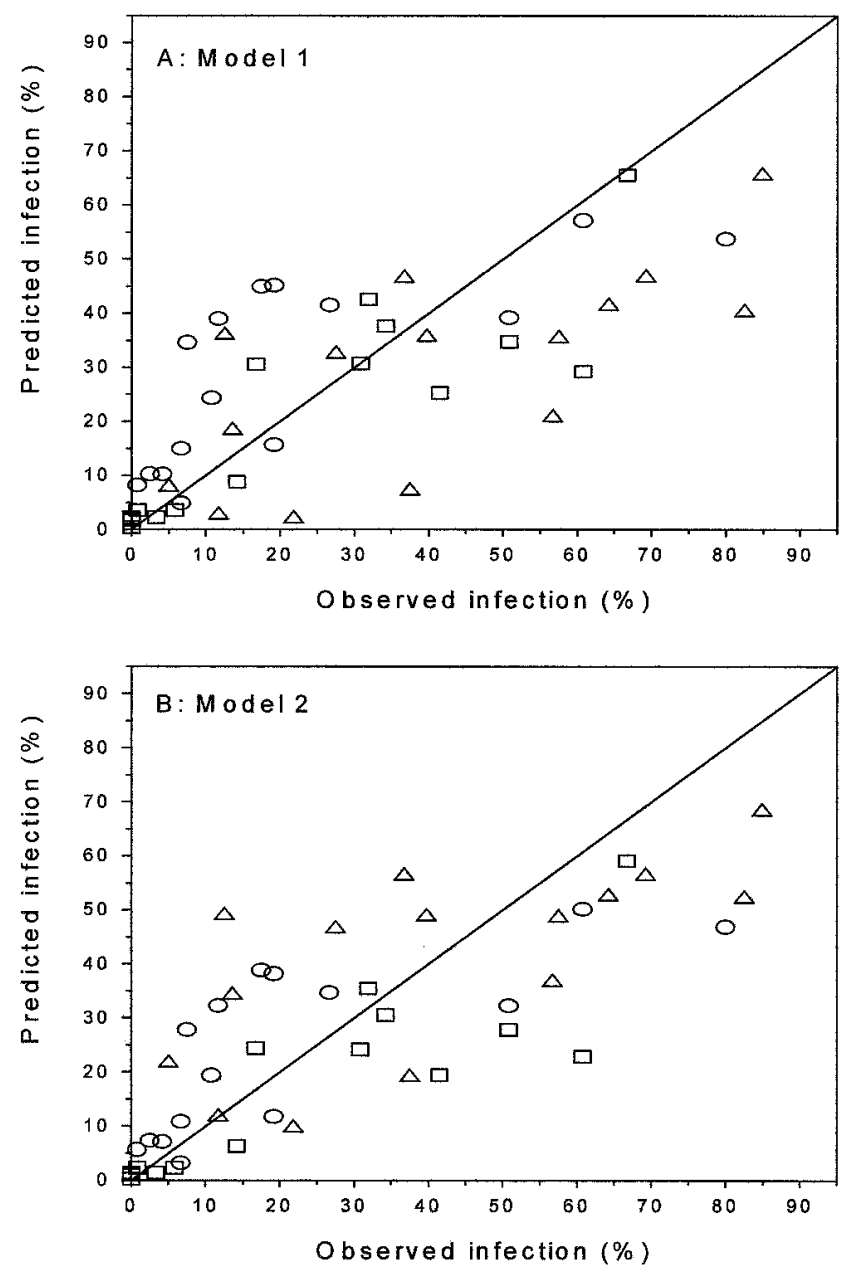

Fig. 4. Comparison of the fitted and the observed incidence of flower infection in each 48-h-period for the models using inoculum only: $\bigcirc=1995 ; \Delta=$ 1996; $\square=1997$. If a predicted incidence is equal to the observed, then this point should be on the solid line. Model parameter estimates are in Table 2. $\left(9.4^{\circ} \mathrm{C}\right)$ and highest in $1995\left(12.3^{\circ} \mathrm{C}\right)$; rainfall was lowest in 1995 $\left(0.83 \mathrm{~mm} \mathrm{day}{ }^{-1}\right)$ and highest in $1996\left(1.98 \mathrm{~mm} \mathrm{day}^{-1}\right)$; vpd was lowest in $1996(2.4 \mathrm{~mm} \mathrm{Hg})$ and highest in $1995(3.8 \mathrm{~mm} \mathrm{Hg})$. The temporal patterns of these variables also differed considerably among years (Figs. 1 through 3 ).

Modeling the number of airborne conidia. TF analysis showed that weather variables accounted for about 68,70 , and $64 \%$ of the total variation in the number of airborne conidia for 1995, 1996, and 1997, respectively. The relationships identified by $\mathrm{TF}$ analysis between weather variables and number of conidia, however, differed significantly between years. For 1995, the number of conidia was correlated positively with wet-moist conditions 11 days previously and negatively with current wet-moist conditions. For 1996, it was correlated positively with wet-moist conditions 8 days previously and negatively with current wet-moist conditions. For 1997, it was correlated positively with wet-moist conditions 3 days previously and with current temperature. In addition, different variables were used to represent wet-moist conditions, e.g., the amount or duration of rainfall or duration of wetness or $r h$. Therefore, it was not possible to derive a simple and consistent equation to relate the daily numbers of conidia to weather variables for the 3 years.

Modeling flower infection using inoculum only. Two alternative models described the relationship between DII and inoculum concentration. In both models, logit $\left(D I I_{t}\right)$ was a linear function of the daily inoculum concentration

$$
\ln \left[D I I_{t} /\left(1-D I I_{t}\right)\right]=f\left(i_{t}\right)=a i_{t}+b
$$

where $a$ and $b$ are parameters. Model 1 had common parameter values for $a$ and $b$ for the 3 years. In contrast, parameters $a$ and $b$ for 1996 differed from those in the other 2 years in model 2, suggesting that the response to inoculum concentration was less in 1996 than in 1995 and 1997 (Table 2). The variation accounted for by Model $2(72 \%)$ was slightly greater than that for model 1 $(64 \%)$, as was the correlation between the fitted and observed incidence of infection ( 0.78 and 0.74 , respectively). The two models appeared to underestimate incidence when the observed incidence was more than $50 \%$ (Fig. 4).

Modeling flower infection using weather variables only. Four different models were derived to relate DII to weather variables only; two of these differentiated between daytime-nighttime values (models 3 and 4) whereas the other two used average daily values (models 5 and 6 ). In all four models, logit $\left(D I I_{t}\right)$ was a linear function of several weather variables.

In models 3 and 4 , three day-night weather variables were included; daytime vpd $\left(d v_{t}\right)$, nighttime temperature $\left(n t_{t}\right)$, and total duration of the surface wetness the previous night $\left(n w_{t-1}\right)$ :

$$
\ln \left[D I I_{t} /\left(1-D I I_{t}\right)\right]=f\left(w_{t}\right)=a d v_{t}+b n t_{t}+c n w_{t-1}+d
$$

\begin{tabular}{|c|c|c|c|c|c|}
\hline \multirow[b]{3}{*}{ Term } & \multirow[b]{3}{*}{ Parameter } & \multicolumn{2}{|c|}{ Day-night variables } & \multicolumn{2}{|c|}{ Daily variables } \\
\hline & & Model 3 & Model 4 & Model 5 & Model 6 \\
\hline & & Common model & $(1995+1997)$ vs. 1996 & Common model & $(1995+1997)$ vs. 1996 \\
\hline Constant $(1995$ + 1997) & $d$ & & $-5.34 \pm 0.883$ & & $-7.37 \pm 0.874$ \\
\hline Constant (1996) & $d$ & $-3.78 \pm 0.727$ & $-1.98 \pm 0.935$ & $-5.33 \pm 0.622$ & $-3.45 \pm 0.824$ \\
\hline Vpd & $a$ & $-0.28 \pm 0.067$ & $-0.25 \pm 0.065$ & $-0.96 \pm 0.147$ & $-0.95 \pm 0.145$ \\
\hline Temperature $(1995$ + 1997) & $b$ & & $0.57 \pm 0.093$ & & $0.67 \pm 0.089$ \\
\hline Temperature (1996) & $b$ & $0.41 \pm 0.067$ & $0.24 \pm 0.086$ & $0.51 \pm 0.066$ & $0.37 \pm 0.076$ \\
\hline Duration of wetness previous night (h) & $c$ & $-0.12 \pm 0.051$ & $-0.13 \pm 0.048$ & & \\
\hline Variation accounted for $(\%)$ & & 58.4 & 63.9 & 57.7 & 65.1 \\
\hline Correlation between fitted and observed & & 0.84 & 0.87 & 0.81 & 0.84 \\
\hline
\end{tabular}

TABLE 3. Multiple regression analyses of the incidence of Botrytis cinerea infection of strawberry flowers on weather variables ${ }^{\text {a }}$

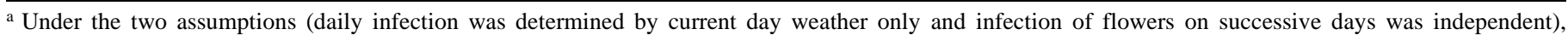
regression results of equation 6 can be interpreted on the basis of $D I I$ (equation 3 ). Thus, the logit of the incidence of daily flower infection [logit $\left.\left(D I I_{t}\right)\right]$ was a function of weather variables, i.e., $\ln \left[D I I_{t} / 1-D I I_{t}\right]=a v p d_{t}+b$ temperature $t_{t}+c$ wetness $_{t-1}+d$ where $a, b, c$, and $d$ are parameters. Average day-night values for weather variables were used in models 3 and 4, whereas average daily values for weather variables were used in models 5 and 6 . Models 3 and 5 had common parameter values for $b$ and $d$ for the 3 years, whereas parameters $b$ and $d$ for 1996 differed from those in the other 2 years in models 4 and 6 . 
where $a, b, c$, and $d$ are parameters. In model 3 , all parameter estimates were assumed to be the same for the 3 years, whereas in model 4 , the parameters $b$ and $d$ were estimated separately for 1996 from the other 2 years (Table 3 ).

The contribution to the model fit by $n w_{t-1}$ was the smallest of the three variables, accounting for only about $5 \%$ of the total variation in $p_{t}$. DII was positively related to night temperature and negatively to daytime vpd and duration of wetness the previous night. The response of $D I I_{t}$ to night temperature was greater in 1995 and 1997 than in 1996, as indicated by model 4. The correlation between fitted and observed incidence was high (0.84 and 0.87 for models 3 and 4, respectively). Except for one point predictions given by models 3 and 4 were very similar (Fig. 5).
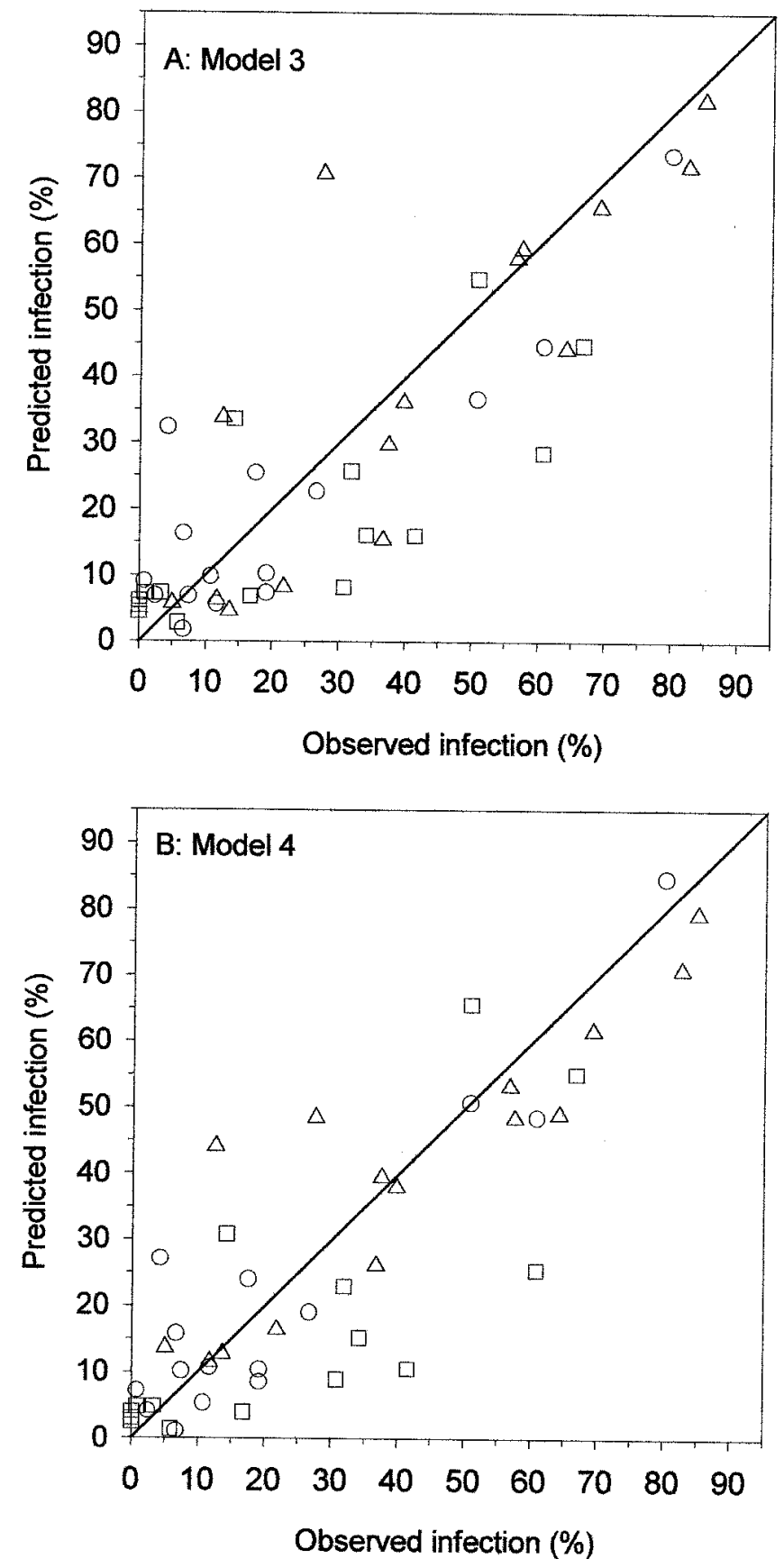

Fig. 5. Comparison of the fitted and the observed incidence of flower infection in each 48-h-period for the models using day-night weather variables only: $O=1995 ; \Delta=1996 ; \square=1997$. If a predicted incidence is equal to the observed, then this point should be on the solid line. Model parameter estimates are in Table 3.
In models 5 and 6, two daily weather variables were included: daily average vpd $\left(v p d_{t}\right)$ and temperature $\left(t p_{t}\right)$, i.e.,

$$
\ln \left[D I I_{t} /\left(1-D I I_{t}\right)\right]=f\left(w_{t}\right)=a v p d_{t}+b t p_{t}+d
$$

where $a, b$, and $d$ are parameters. In model 5 , all parameter estimates were the same for the 3 years, whereas in model 6 , the parameter $b$ was estimated separately for 1996 from the other 2 years (Table 3 ).

Daily incidence of infection was related positively to daily average temperature and negatively to daily average vpd. The response of $D I I_{t}$ to temperature was greater in 1995 and 1997 than in 1996 , as indicated by model 6 . The correlation between fitted and the observed incidence was similarly high, i.e., 0.81 and 0.84 for models 5 and 6, respectively. Predictions given by both models appeared to be less accurate than those given by models 3 and 4 . As an example, the combined effects of vpd and temperature on the incidence of flower infection (calculated using model 5) are shown in Figure 6.

Modeling flower infection using both weather and inoculum variables. Four different models were used to relate DII to weather and inoculum variables. Two used daytime-nighttime values of weather variables (models 7 and 8), and two used average daily values (models 9 and 10).

Models 7 and 8 included two weather variables in addition to inoculum: daytime vpd $\left(d v_{t}\right)$ and nighttime temperature $\left(n t_{t}\right)$ :

$$
\ln \left[D I I_{t} /\left(1-D I I_{t}\right)\right]=f\left(w_{t}\right) i_{t}=\left(a d v_{t}+b n t_{t}\right) i_{t}+c
$$

where $a, b$, and $c$ are parameters. In model 7, all parameter estimates were the same for the 3 years, whereas in model 8 parameters $b$ and $c$ were estimated separately for 1996 from the other 2 years (Table 4). DII was positively related to night temperature and negatively to daytime vpd. The correlation between fitted and observed incidence was high for models 7 and 8 (0.86 and 0.89, respectively). Values fitted by model 7 were very similar to those fitted by model 8 (Fig. 7).

In addition to inoculum, two daily weather variables were included in models 9 and 10: daily average vpd $\left(v p d_{t}\right)$ and temperature $\left(t p_{t}\right)$ :

$$
\ln \left[D I I_{t} /\left(1-D I I_{t}\right)\right]=f\left(w_{t}\right) i_{t}=\left(a v p d_{t}+b t p_{t}\right) i_{t}+c
$$

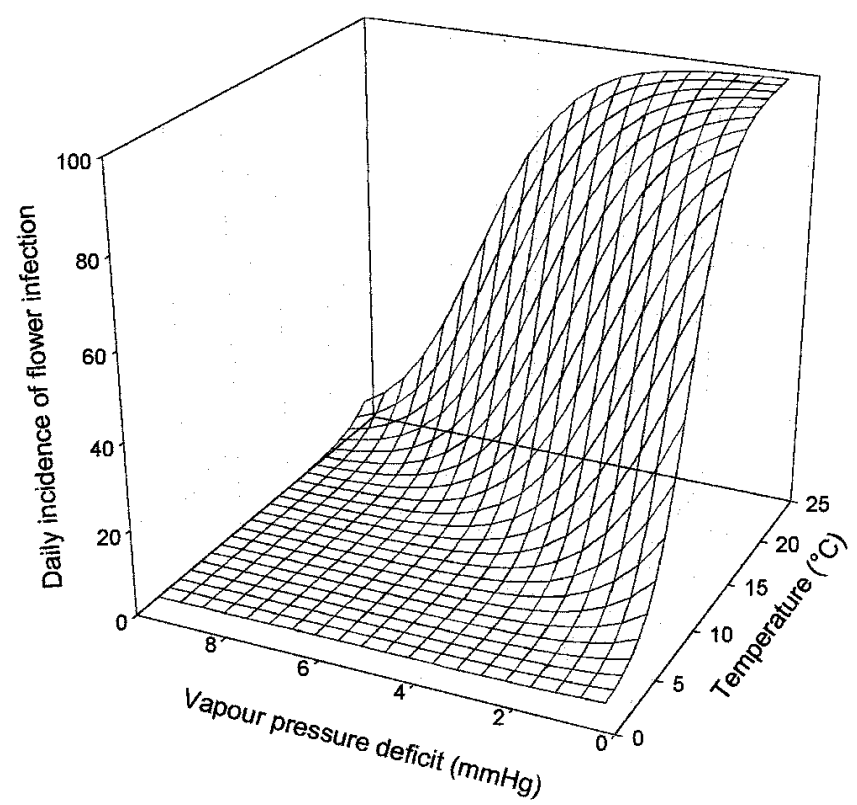

Fig. 6. Estimated relationship of daily incidence of infection of strawberry flowers by Botrytis cinerea to daily average vapor pressure deficit and temperature (model 5). Model 5 parameter estimates are given in Table 3. 
where $a, b$, and $c$ are parameters. In model 9, all parameter estimates were the same for the 3 years. In model 10, parameters $b$ and $c$ were estimated separately for 1996 from the other 2 years (Table 4). Daily infection incidence was positively related to daily average temperature and negatively to daily average vpd. The response of $D I I_{t}$ to temperature was greater in 1995 and 1997 than in 1996. The correlation between fitted and observed incidence was 0.86 and 0.88 for models 9 and 10 , respectively.

\section{DISCUSSION}

Analysis of data obtained from unsprayed field plots over 3 years showed a strong relationship between incidence of flower infection by $B$. cinerea and weather conditions and inoculum con-
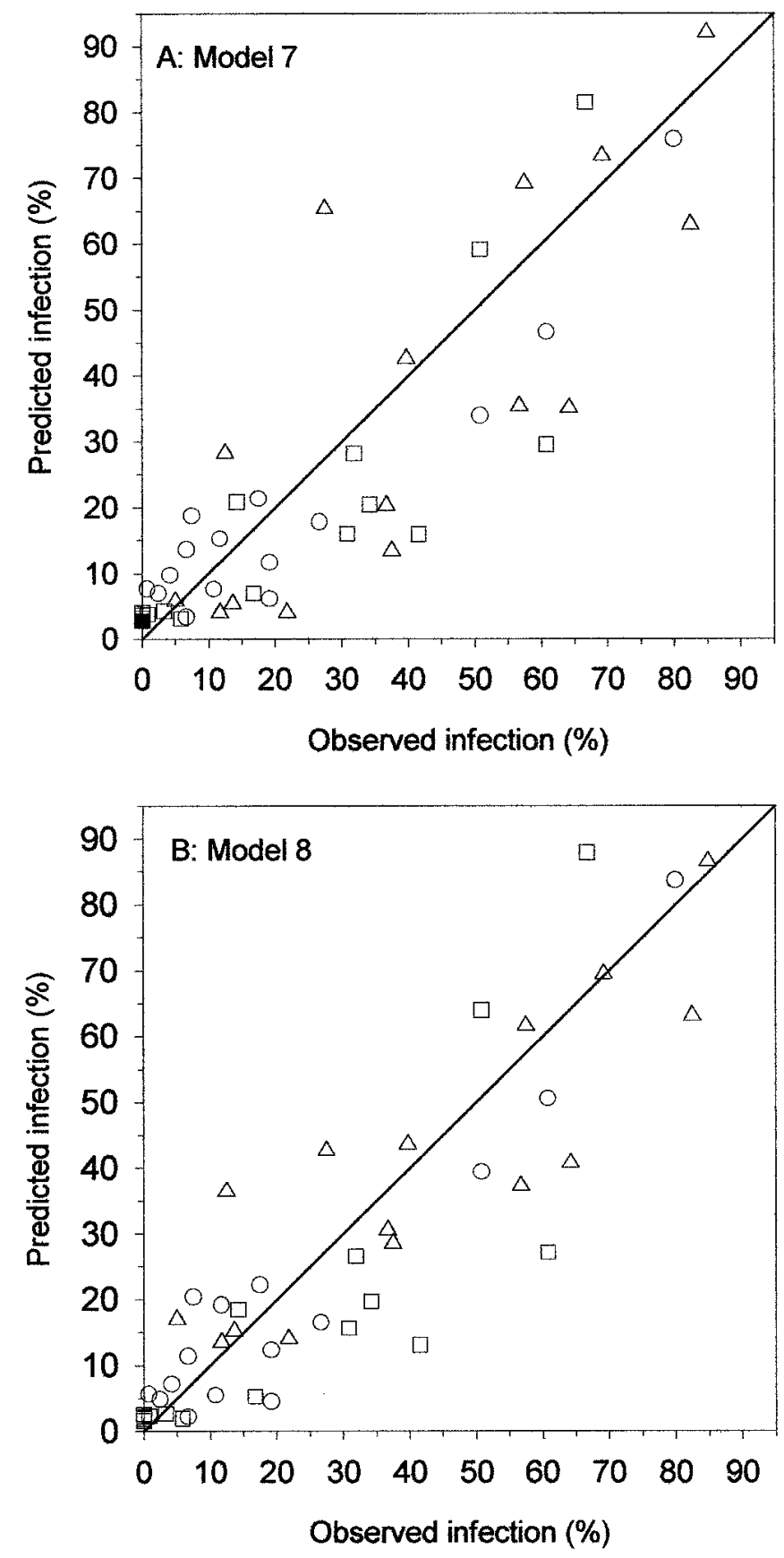

Fig. 7. Comparison of the fitted and the observed incidence of flower infection in each 48-h-period for the models using both inoculum and day-night weather variables: $\bigcirc=1995 ; \Delta=1996 ; \square=1997$. If a predicted incidence is equal to the observed, then this point should be on the solid line. Model parameter estimates are given in Table 4. centration measured as the number of conidia in the air over the crop. Models based on various combinations of weather variables and inoculum concentration satisfactorily predicted infection incidence, and moreover, the models were consistently accurate over 3 years. As expected, the incidence of flower infection was positively correlated with inoculum concentration. Vapor pressure deficit and temperature were the two most important weather variables influencing flower infection. Increasing temperature and decreasing vpd resulted in higher incidences of flower infection. The effects of weather variables in this study are consistent with the results of other studies on the epidemiology of gray mold. For example, the incidence of gray mold of fruit was positively correlated with a long duration of high humidity during flowering (22).

The effects of inoculum concentration and temperature on the infection of flowers in 1996 were significantly different from those in 1995 and 1997 despite the fact that inoculum concentration in 1996 was higher than in 1995 but less than in 1997. The differential responses of flower infection to inoculum and temperature were relatively small, however, and appeared to be confined to only a few points. Moreover, overall prediction accuracy was similar between the two sets of models. The high degree of consistency in models between years suggests that models based on common parameter estimates should provide satisfactory predictions of the risk of flower infection.

Most previous work on the epidemiology and control of gray mold has been concerned with the effects of environmental variables and fungicide application and timing on the subsequent incidence of the disease. The level of inoculum in general has not been explicitly considered in these studies. In controlled laboratory studies, plant tissues are usually inoculated with high doses of inoculum and then subjected to various combinations of wetness duration and temperature $(2,4,16)$. Models or prediction systems developed from such studies do not usually take into account variable levels of inoculum under field conditions, which may affect their accuracy in predicting disease in field conditions. This study shows that including inoculum in the models in addition to weather variables improved model performance although only slightly. Furthermore, the models based only on weather variables gave more accurate predictions than the models using inoculum only.

Despite being developed from field data, the models developed in this study are simple and generally consistent. The model variables have clear biological interpretations. Vapor pressure deficit and temperature were identified as the two key variables in determining the incidence of flower infection, especially vpd during the day and temperature at night. This is consistent with the biology of $B$. cinerea; a long duration of high humidity (wetness) and increasing temperature (below approximately $20^{\circ} \mathrm{C}$ ) lead to high infection incidence $(4,13,22)$. During the day when most conidia are released and dispersed (10), the limiting factor for conidial survival and infection is most likely to be atmospheric moisture content measured by vpd. The higher the vpd, the less favorable conditions are for conidial survival and infection (5). Atmospheric moisture content is generally very high and near saturation on spring nights but temperature is generally low, often below $10^{\circ} \mathrm{C}$. Consequently, the limiting factor for infection is likely to be temperature. The higher the temperature (below $20^{\circ} \mathrm{C}$ ), the higher the incidence of infection $(4,21)$. In the models that depend on day-night variables (models 3 and 4), duration of wetness during the previous night had small but significant negative effects on the incidence of flower infection. This is probably due indirectly to its negative effects on conidial dispersal. This is further supported by the fact that nighttime wetness did not improve the fit of the models that included the inoculum factor. In the present study, the observed infection was assumed to have occurred during the period from white bud (tagging time) to open flowers (collecting time), and infection of flowers at green bud stages was ignored. The resulting models, therefore, may 
overestimate the effects of daily weather variables on the infection of flower parts. Such an overestimation, however, should be small because flowers at the open, white-bud, and senescent stages are much more susceptible to infection than those at greenbud stage $(9,14)$.

Even though flower sampling in the present study was done at 48-h-intervals, data analyses were based on a convenient, discrete daily period, which was necessary to represent continuous epidemic processes, such as spore production and dispersal, and subsequent infection. The validity of these models depends on the two assumptions used for data analysis, which are necessary to deduce the incidence of daily infection from the observed incidence over 2-day periods. Infection of flowers on successive days was assumed to be independent. The success of infection by conidia released on any day was assumed to be affected only by weather conditions on the same day. This assumption is based on the fact that most conidia of $B$. cinerea are released between early morning and early afternoon (10). Success of flower infection by conidia depends critically on weather conditions during the first 16 to $24 \mathrm{~h}$ after deposition of conidia on flower surfaces. However, conidia are also released in great numbers during rainfall (13). This pattern of conidial dispersal, especially if rainfall occurs during the late afternoon and night, may invalidate this assumption. One possible consequence of this is an underestimation of the effects of wetness on infection. Nevertheless, the performance and consistency of the models over several years and the biological interpretations of each model variable indicate that these two assumptions are justified. Sampling in 24-h-intervals might improve the models, but intensive sampling was impractical for such a long sampling period in each of the 3 years.

Detailed studies of infection of strawberry flowers by $B$. cinerea showed that stamens may be an important avenue for latent fruit infection (1). In the present study, most of the infection recorded was on petals. Recording infection over a 48-h period from white bud may have underestimated the contribution of stamen infection to final fruit infection. Nevertheless, the incidence of fruit rot originating from infected flowers was more closely related to the incidence of petal infection by $B$. cinerea conidia rather than the incidence of stamen infection $(4,21)$. In the first $24 \mathrm{~h}$ of each 48 -h sampling period, flowers were at the white bud stage, while in the second 24-h period flowers were mostly in the open-flower stage. Although flowers at both white-bud and openflower stages are highly susceptible, infections at the white-bud stage are much less likely to lead to gray mold than infections at the open-flower stage (14). Thus, the incidence of petal infection observed in this study might overestimate potential incidence of gray mold originating from flower infections. Currently, work is being conducted to investigate whether the present models could be used to predict fruit infection under field conditions.

The models from this study could probably be more easily adopted for use in field conditions than models based on data obtained from laboratory studies. Laboratory-based models usually rely on the duration of wetness and temperature during the wetness period. Definition of surface wetness is often problematic and its measurement needs an automatic data logger, but there is no single accepted industry standard in the design of wetness sensors. By contrast, wetness duration is not essential in the models described here and the two key variables for these models can be obtained using simple, low-cost instruments. Secondly, when using laboratory-based models that include surface wetness duration, it is necessary to include a threshold duration of dry periods to terminate a wet period (or infection period). This value is very difficult to obtain.

The models developed in the present study only predict daily incidence of infection of strawberry flowers by $B$. cinerea. Although conditions required for infection of ripe fruit are less stringent than for infection of flower parts (3), it is generally accepted that infection of strawberry flowers and the subsequent establishment of quiescent mycelia in flower parts is the main avenue leading to rotting of ripe fruit at and after harvest $(9,11,12,14,17)$. The incidence of fruit rot originating from infected flowers can be estimated from the incidence of petal infection using an existing model $(4,21)$. To help growers in timing fungicide applications, it may be necessary to set a threshold value for incidence of flower infections. Incidence of fruit rot increased very little with the incidence of petal infection until petal infection exceeded $50 \%(4,21)$, at which point the incidence of fruit rot was estimated at $11 \%$. Therefore, $50 \%$ of daily petal infection could be used as a very conservative threshold. Assuming that most flowers open in a period of 20 days, a threshold of $50 \%$ of daily petal infection $(\approx 11 \%$ infection of fruits from those open flowers on that particular day) is equivalent to $0.55 \%$ of total fruit infected. Currently, the models are being evaluated under field conditions on both short-day and day-neutral types of strawberry to assess their effectiveness in guiding spray application timing. Preliminary evaluation results on the short-day type of strawberry showed that by timing the fungicide application according to model predictions a reduction of $60 \%$ fungicide input was possible while maintaining satisfactory control of gray mold compared with the routine spray program. The effects of weather conditions and inoculum on infection of fruit are currently under investigation using an experimental approach similar to the one used in this study. These results, together with those obtained in this study, will be the basis of an integrated management system for gray mold.

TABLE 4. Multiple regression analyses of the incidence of Botrytis cinerea infection of strawberry flower on daily inoculum (daily number of conidia trapped) and weather variables ${ }^{\mathrm{a}}$

\begin{tabular}{|c|c|c|c|c|c|}
\hline \multirow[b]{3}{*}{ Term } & \multirow[b]{3}{*}{ Parameter } & \multicolumn{2}{|c|}{ Day-night weather variables } & \multicolumn{2}{|c|}{ Daily weather variables } \\
\hline & & Model 7 & Model $8^{a}$ & Model 9 & Model 10 \\
\hline & & Common model & $(1995+1997)$ vs. 1996 & Common model & $(1995+1997)$ vs. 1996 \\
\hline Constant (1996) & $c$ & $-4.34 \pm 0.327$ & $-2.71 \pm 0.498$ & $-4.47 \pm 0.336$ & $-2.72 \pm 0.487$ \\
\hline Vapor pressure deficit & $a$ & $-0.27 \pm 0.073$ & $-0.27 \pm 0.070$ & $-0.88 \pm 0.152$ & $-0.88 \pm 0.145$ \\
\hline Temperature $(1995+1997)$ & $b$ & & $0.57 \pm 0.060$ & & $0.57 \pm 0.056$ \\
\hline Temperature (1996) & $b$ & $0.48 \pm 0.050$ & $0.31 \pm 0.063$ & $0.49 \pm 0.049$ & $0.33 \pm 0.056$ \\
\hline
\end{tabular}

${ }^{a}$ Under the two assumptions (daily infection was determined by current-day weather only and infection of flowers on successive days was independent), regression results of equation 6 can be interpreted on the basis of $D I I$ (equation 3). The logit of the incidence of daily flower infection (logit $\left(D I I_{t}\right)$ ) was a function of weather variables and daily inoculum. A multiplicative relationship was assumed between inoculum and weather variables, i.e., all the weather variables were multiplied by the relative number of conidia trapped on the same day: $\left.\ln \left(D I I_{t} / 1-D I I_{t}\right)=\left(a v p d_{t}+b \text { temperature }\right)_{t}\right) i_{t}+c$ where $a, b$, and $c$ are parameters. Average day-night values of weather variables were used in models 7 and 8 , whereas average daily values of weather variables were used in models 9 and 10. Models 7 and 9 had common parameter values for $b$ and $c$ for the three years, whereas parameters $b$ and $c$ for 1996 differed from those in the other 2 years in models 8 and 10 . 


\section{ACKNOWLEDGMENTS}

This work was funded by the British Ministry of Agriculture, Fisheries, and Food (MAFF).

\section{LITERATURE CITED}

1. Bristow, P. R., McNicol, R. J., and Williamson, B. 1986. Infection of strawberry flowers by Botrytis cinerea and its relevance to gray mould development. Ann. Appl. Biol. 109:545-554.

2. Broome, J. C., English, J. T., Latorre, B. A., and Aviles, J. C. 1995. Development of an infection model for Botrytis bunch rot of grapes based on wetness duration and temperature. Phytopathology 85:97-102.

3. Bulgar, M. A. 1986. The influence of temperature and wetness duration on the infection of strawberry flowers and fruit by Botrytis cinerea. M.S. thesis, Ohio State University, Wooster.

4. Bulger, M. A., Ellis, M. A., and Madden, L. V. 1987. Influence of temperature and wetness duration on infection of strawberry flowers by Botrytis cinerea and disease incidence of fruit originating from infected flowers. Phytopathology 77:1225-1230.

5. Coley-Smith, J. R. 1980. Sclerotia and other structures in survival. Pages 85-115 in: The Biology of Botrytis. J. R. Coley-Smith, K. Verhoeff, and W. R. Jarvis, eds. Academic Press, London.

6. Elad, Y. 1994. Biological control of grape grey mould by Trichoderma harzianum. Crop Prot. 13:35-38.

7. Elad, Y. 1996. Mechanisms involved in the biological control of Botrytis cinerea incited diseases. Eur. J. Plant Pathol. 102:719-732.

8. Ellis, M. A., Madden, L. V., Dale, A., and Luby, J. J. 1991. Progress in the development of disease forecasting systems for strawberry fruit rots in Ohio. Pages 244-251 in: The Strawberry into the 21st Century. Proc. 3rd N. Am. Strawberry Conf. Timber Press, Singapore.

9. Hennebert, G. L., and Gilles, G. L. 1958. Epidemiologie de Botrytis cinerea Pers. sur frasiers. Meded. Landbouwhogesch. Opzoekingsstn
Staat. Gent 23:864-888.

10. Jarvis, W. R. 1962. The dispersal of spores of Botrytis cinerea Fr. in a raspberry plantation. Trans. Br. Mycol. Soc. 45:549-559.

11. Jarvis, W. R. 1962. The infection of strawberry and raspberry fruits by Botrytis cinerea Fr. Ann. Appl. Biol. 50:569-575.

12. Jarvis, W. R. 1964. The effect of some climatic factors on the incidence of grey mould of strawberry and raspberry fruit. Hortic. Res. 3:65-71.

13. Jarvis, W. R. 1980. Epidemiology. Pages 219-250 in: The Biology of Botrytis. J. R. Coley-Smith, K. Verhoeff, and W. R. Jarvis, eds. Academic Press, London.

14. Jarvis, W. R., and Borecka, H. 1968. The susceptibility of strawberry flowers to infection by Botrytis cinerea Pers. ex Fr. Hortic. Res. 8:147154.

15. Kaye, G., and Laby, T. 1957. Tables of physical and chemical constants and some mathematical functions. 11th ed. Longmans, Green and Co., London.

16. Nair, N. G., and Allen, R. N. 1993. Infection of grape flowers and berries by Botrytis cinerea as a function of time and temperature. Mycol. Res. 97:1012-1014.

17. Powelson, R. L. 1960. Initiation of strawberry fruit rot caused by Botrytis cinerea. Phytopathology 50:491-494.

18. Sutton, J. C. 1994. Biological control of strawberry disease. Adv. Strawberry Res. 13:1-12.

19. Sutton, J. C., and Peng, G. 1993. Biocontrol of Botrytis cinerea in strawberry leaves. Phytopathology 83:615-621.

20. Wei, W. W. S. 1990. Time series analysis: Univariate and Multivariate Methods. Addison-Wesley, New York.

21. Wilcox, W. F. 1989. Influence of environment and inoculum density on the incidence of brown rot blossom blight of sour cherry. Phytopathology 79:530-534.

22. Wilcox, W. F., and Seem, R. C. 1994. Relationship between strawberry gray mold incidence, environmental variables, and fungicide applications during different periods of the fruiting season. Phytopathology $84: 264-270$ 